\title{
Management algorithms for prostate-specific antigen progression in prostate cancer: Biochemical recurrence after definitive therapy and progression to non-metastatic castrate-resistant prostate cancer
}

\author{
Brita Danielson ${ }^{1}$; Fred Saad ${ }^{2}$; Alan So ${ }^{3}$; Scott Morgan ${ }^{4}$; Robert J. Hamilton ${ }^{5}$; Shawn Malone ${ }^{4}$ \\ Laura Park-Wyllie $^{6}$; Anousheh Zardan ${ }^{6}$; Bobby Shayegan ${ }^{7}$ \\ ${ }^{1}$ Cross Cancer Institute, University of Alberta, Edmonton, AB, Canada; ${ }^{2}$ Centre Hospitalier de l'Université de \\ Montréal, University of Montreal, Montreal, QC, Canada; ${ }^{3}$ Vancouver Prostate Centre, University of British \\ Columbia, BC, Canada; ${ }^{4}$ The Ottawa Hospital, University of Ottawa, Ottawa, ON, Canada; ${ }^{5}$ Princess Margaret \\ Cancer Centre, University of Toronto, Toronto, ON, Canada; ${ }^{6}$ Medical Affairs, Janssen Inc., Toronto, ON, \\ Canada; ${ }^{7}$ Juravinski Cancer Centre, McMaster University, Hamilton, ON, Canada
}

Funding: This article was prepared according to ICMJE recommendations, with editorial and research assistance from Kaleidoscope Strategic Inc. Janssen Canada supported the development of the algorithm, provided funding for medical writing services, and their employees were contributing authors. Full COI disclosures will be provided in the final published version.

Acknowledgements: The authors would like to thank Janssen Canada for funding this initiative, Drs. Geoffrey Gotto, Naveen Basappa, Henry Conter, Jeffrey Spodek, Lorne Aaron, Kim Chi, Sebastien Hotte, Tony Finelli, and Christina Canil for their contributions to algorithm development, and Deanna McLeod, Ilidio Martins, and Loretta Collins of Kaleidoscope Strategic Inc. for research and editorial support in preparing the manuscript.

Cite as: Can Urol Assoc J 2019 February 7; Epub ahead of print. http://dx.doi.org/10.5489/cuaj.5600

Published online February 7, 2019

$* * *$

\section{Abstract}

Introduction: Current prostate cancer (PC) guidelines primarily focus on localized or metastatic PC. A multidisciplinary genitourinary oncology panel determined that additional guidance focusing on monitoring and management of biochemical recurrence (BCR) following radical therapy and non-metastatic castration-resistant prostate cancer (nmCRPC) was warranted. Methods: The most up-to-date national and international guidelines, consensus statements, and emerging phase 3 trials were identified and used to inform development of algorithms by a multidisciplinary genitourinary oncology panel outlining optimal monitoring and treatment for patients with non-metastatic PC. 
Results: A total of eight major national and international guidelines/consensus statements published since 2015 and three phase 3 trials were identified. Working group discussions among the multidisciplinary genitourinary oncology panel led to the development of two algorithms: the first addressing management of patients with BCR following radical therapy (post-BCR), and the second addressing management of nmCRPC. The post-BCR algorithm suggests consideration of early salvage treatment in select patients, and provides guidance regarding observation vs. intermittent or continuous androgen-deprivation therapy (ADT). The nmCRPC algorithm suggests continued ADT and monitoring for all patients, with consideration of treatment with apalutamide or enzalutamide for patients with high-risk disease (prostate-specific antigen [PSA] doubling time of $\leq 10$ months).

Conclusions: Two treatment algorithms have been developed to guide the management of nonmetastatic PC, and should be considered in the context of local guidelines and practice patterns.

\section{Introduction}

Prostate cancer (PC) is the most common cancer diagnosis in Canadian men, with an estimated 21,300 new cases in 2017. ${ }^{1}$ Following radical prostatectomy (RP) and/or radiation therapy (RT), up to $27-53 \%$ will experience a biochemical recurrence (BCR), ${ }^{2,3}$ defined as prostate-specific antigen (PSA) $>0.2 \mathrm{ng} / \mathrm{ml}$ following RP, or a PSA nadir $+2 \mathrm{ng} / \mathrm{ml}$ after RT..$^{3-7}$ Many of these patients will then progress to non-metastatic castration-resistant prostate cancer (nmCRPC), defined as no visible metastases on conventional imaging and a rising PSA despite a castrate testosterone level. This is sometimes also referred to as m0CRPC.

Although national and international guidelines and consensus statements provide guidance on the management of PC, recommendations are predominantly focused on localised or metastatic disease, ${ }^{3,4,8-13}$ with less in-depth consideration of monitoring and management of post-BCR and nmCRPC patients. ${ }^{3,4,6,7,14}$ Standard therapies after BCR include local salvage therapy, androgen deprivation therapy (ADT) and observation ${ }^{3,4,15}$ and, until recently, treatment for nmCRPC consisted of continued ADT, secondary hormonal manipulations, observation and monitoring, or clinical trials. ${ }^{3,4,6,14,16}$ With the advent of new systemic therapies for nmCRPC, ${ }^{17}$, ${ }^{18}$ more detailed guidance on optimal treatment for patients with BCR after radical therapy and progression to nmCRPC is warranted.

A group of Canadian multidisciplinary genitourinary specialists identified a significant gap in guidance regarding the-management of BCR and subsequent nmCRPC. They therefore set out to develop practical algorithms for these disease states, informed by the most recent phase III data, national and international guidance, and Canadian multidisciplinary clinical expert opinion. 


\section{Methods}

\section{Algorithm development}

The panel comprised five uro-oncologists, three radiation oncologists, two medical oncologists and three medical advisors from Janssen, and had pan-Canadian representation. The group held iterative discussions regarding the management of patients with BCR after radical therapy and nmCRPC. Algorithms outlining monitoring and treatment sequencing were drafted and further refined through review of national and international guidelines, consensus statements, and emerging phase III data (see Literature Search below).

\section{Literature search}

Guidelines and consensus statements were leveraged to identify current guidance on the management of non-metastatic PC. PubMed, Google Scholar and web-based searches were carried out for Canadian, North American and European guidelines or consensus statements drafted or updated from January 2015 to March 26, 2018. Articles of interest were identified using the search strings prostate cancer AND (guideline OR consensus OR recommendations) AND (biochemical recurrence OR biochemical failure OR castrate-resistant).

As existing guidelines were current to March 2018 (NCCN 2018 v2; data cut-off February 2018), ${ }^{4}$ a supplementary search for new data was performed. PubMed and ASCO GU were searched for reports of original phase III trials on management of BCR after local radical therapy and non-metastatic PC, published or presented from January 2018 to April 5, 2018, using the following keywords: prostate cancer AND biochemical recurrence OR castrate-resistant (OR respective aliases). Search results were screened at the abstract level and studies of interest were confirmed at full text.

\section{Results}

\section{Literature search findings and algorithm development}

Two pan-Canadian guidelines or consensus statements ${ }^{14,15}$ and six guidelines or consensus statements from major North-American and/or European organizations or groups 3, 4, 6, 7, 12, 16 published since 2015 were identified (Table 1), along with one new monitoring study for BCR after radical therapy, ${ }^{19}$ and two studies on new treatments for nmCRPC (Table 1). ${ }^{17}{ }^{18}$ Canadian provincial guidelines, ${ }^{8-10}$ European single-nation ${ }^{11,20-22}$ and specialty PC guidelines ${ }^{23}$ were excluded. The iterative group discussion process and review of national and international guidance led to the development of two algorithms: the first addressing monitoring and management of patients post-BCR (Fig. 1), and the second addressing management of nmCRPC (Fig. 2).

\section{Monitoring and treatment after biochemical recurrence but before progression to nmCRPC}


Definitions of BCR and local salvage treatment options depend on the type of therapy received for localized disease. Following RT, BCR is defined as PSA level of $2 \mathrm{ng} / \mathrm{ml}$ or more above the PSA nadir achieved following therapy (PSA nadir $+2 \mathrm{ng} / \mathrm{ml}$; Fig. 1 ). ${ }^{3-5}$ The definition of BCR in patients after RP is PSA $>0.2 \mathrm{ng} / \mathrm{ml}$ (Fig. 1). ${ }^{3,4,6,7}$

Post-RT salvage therapy

EAU guidelines recommend local salvage therapy after RT via surgery (i.e., salvage RP), while NCCN recommendations include surgery, brachytherapy or cryotherapy in patients with limited initial disease (T1-T2), a PSA $<10 \mathrm{ng} / \mathrm{ml}$, localized disease (NX-N0) and a reasonable life expectancy ( $>10$ years). ${ }^{3,4}$ To ensure the earliest possible referral, the panel suggests a PSA threshold $<5 \mathrm{ng} / \mathrm{ml}$ and biopsy proven local recurrence. ${ }^{3,4}$

Post-RP salvage therapy

It is beneficial to initiate salvage therapy early, when pre-treatment PSA is low. ${ }^{3,4,12}$ EAU, NCCN and ESMO guidelines recommend initiation of salvage RT when PSA becomes detectable (<0.5 ng/ml), ${ }^{3,4,12}$ up to a PSA limit of $2 \mathrm{ng} / \mathrm{ml}$ (25\% agreement, APCCC). ${ }^{7}$ Similarly, to ensure the earliest possible referral, the panel suggests consideration of salvage therapy when PSA becomes detectable $>0.1 \mathrm{ng} / \mathrm{ml}$ (Fig. 1). ${ }^{3}$

Imaging prior to salvage therapy

When a patient experiences a BCR after radical local therapy and salvage therapy is being considered, standard imaging, including computed tomography (CT) of the abdomen and pelvis and a bone scan (BS) are recommended to rule out metastatic disease. Use of magnetic resonance imaging (MRI) may also be indicated if local recurrence after prostatectomy is suspected and salvage therapy is being considered. Novel imaging modalities currently being evaluated, such as ${ }^{18} \mathrm{~F}$-fluciclovine positron emission tomography/computed tomography (PET/CT) and prostatespecific membrane antigen-positron emission tomography (PSMA-PET), may be helpful in staging patients to guide consideration of salvage therapy. ${ }^{19,24,25}$

Other treatment and monitoring options for BCR

Observation and ADT are appropriate management options for patients with BCR who do not undergo salvage therapy, or who experience a BCR despite salvage therapy (Fig. 1). In order to appropriately tailor treatment, the panel suggests regular monitoring consisting of PSA and testosterone every 3-6 months, 3, 4, 15 and imaging via CT and BS when PSA rises rapidly, ${ }^{3,4,7}$ or if PSA is $>20 \mathrm{ng} / \mathrm{ml}$ in the off-treatment period of intermittent ADT (iADT). CT and BS should also be considered if PSA is rising ( $>2 \mathrm{ng} / \mathrm{ml}$ ) despite castrate levels of testosterone, or if a patient is symptomatic.

Both NCCN and EAU guidelines recommend observation in men with prolonged ( $>12$ months) prostate-specific antigen doubling time (PSAdt). ${ }^{3,4}$ The panel suggests observation with 
lower PSA levels ( $<10 \mathrm{ng} / \mathrm{ml}$ after RT; $<5 \mathrm{ng} / \mathrm{ml}$ after RP) and longer PSAdt ( $>10$ months; Fig. 1). Guidance related to administration of ADT, either intermittent or continuous, is limited in guidelines and consensus statements. The panel suggests use of $\mathrm{iADT}^{3,4,7}$ when PSA thresholds are reached $(\mathrm{RT},>10 \mathrm{ng} / \mathrm{ml}$ or $\mathrm{RP},>5 \mathrm{ng} / \mathrm{ml}$, or with PSAdt $\leq 10$ months (Fig. 1). Although most patients respond to initial iADT, ${ }^{26,27}$ switching to continuous ADT (cADT) is suggested if the PSA nadir is $\geq 1 \mathrm{ng} / \mathrm{ml}$ after 6 months of iADT or if the iADT off-treatment interval is $<10$ months (Fig. 1). Achieving lower testosterone levels $(\leq 0.7 \mathrm{nmol} / \mathrm{l})$ has been associated with improved outcomes; therefore secondary hormonal manipulations may also be considered to maintain optimal castrate levels, especially if PSA does not reach a nadir of $<1 \mathrm{ng} / \mathrm{ml}^{15}$

Progression to $\mathrm{nmCRPC}$

Progression to CRPC is defined as a rising PSA despite a testosterone level of $\leq 1.7 \mathrm{nmol} / 1$. ${ }^{3,}$, 14 Management of CRPC varies depending on the presence or absence of metastases, and suggested treatment for patients with nmCRPC, confirmed via CT/BS, is summarized in Figure 2. 3, 4, 6, 7, 14, ${ }^{15}$ Treatment for patients with metastatic CRPC will be discussed in a subsequent publication.

\section{Monitoring and treatment of $\mathrm{nmCRPC}$}

With progression to nmCRPC, discussion of treatment options, including clinical trial eligibility, should be conducted within the context of a multi-disciplinary consultation. ${ }^{3,4,6,12,14}$

Testosterone should be monitored to ensure castrate levels. ${ }^{3,4,15}$ Guidelines and consensus recommendations for monitoring PSA and testosterone while on ADT vary from every 3-6 months for those with low risk disease and slow PSAdt and/or good prior response to ADT, to more intensive schedules for those with a rapidly rising PSA. ${ }^{3,4} 16$ The panel suggests distinct monitoring schedules based on disease risk (Fig. 2). ${ }^{4,7,16-18}$ For high-risk disease, characterized by a PSAdt of $\leq 10$ months or a PSA level $>8 \mathrm{ng} / \mathrm{ml},{ }^{28-30}$ PSA and testosterone should be assayed every 3 months, 3, 4, 7, 15, 16 with CT and BS every 3-6 months or when symptomatic (Fig. 2). 3, 4, 7, ${ }^{12,14}$ For low-risk disease, defined as PSAdt of $>10$ months, PSA and testosterone should be assayed every 3-6 months ${ }^{3,4,7,15,16}$ with CT and BS every 6 months to 1 year, or when symptomatic (Fig. 2). ${ }^{3,4,7,12,14}$

Due to a historical lack of treatment options for nmCRPC, most guidelines published prior to 2018 recommend continued ADT and monitoring with observation for both high-risk and low-risk disease. ${ }^{3,4,12,14}$ Based on recent data from phase III trials and the latest NCCN guidelines, ${ }^{4}$ the panel recommends consideration of the emerging therapies for high risk disease (i.e., apalutamide, Health Canada [HC] approved; enzalutamide, HC approval pending). ${ }^{17,18}$ The recent placebo-controlled SPARTAN ${ }^{18}$ and PROSPER ${ }^{17}$ trials demonstrated that addition of each drug to ADT significantly improved the primary end-point of metastasis-free survival in patients with PSAdt of $\leq 10$ months. Apalutamide prolonged the metastasis-free interval by 24.3 months compared to placebo (40.5 vs. 16.2 months, hazard ratio [HR] 0.28; 95\% confidence interval 
[CI], 0.23 to $0.35 ; \mathrm{P}<0.001),{ }^{18}$ while enzalutamide extended the interval by 21.9 months (36.6 vs. 14.7 months, HR 0.29 ; $95 \%$ CI, 0.24 to 0.35 ; P<0.001). ${ }^{17}$ Although not statistically significant, a trend toward improved overall survival compared to placebo was apparent for both apalutamide (median follow-up of 20.3 months; not reached [NR] vs. 39.0 mo., HR 0.70; 95\% CI, 0.47 to 1.04; $\mathrm{P}=0.07$ ) and enzalutamide (median follow-up of 18.5 months and 15.1 months for enzalutamide and placebo, respectively; NR vs. NR, HR 0.80; 95\% CI, 0.58 to 1.09; $\mathrm{P}=0.15) .{ }^{17}$, 18

Addition of either apalutamide or enzalutamide to ADT led to an increase in adverse events compared to placebo. ${ }^{17,18}$ The rate of treatment discontinuation due to toxicity for apalutamide was $10.6 \%$ compared to $7.0 \%$ for placebo. There were higher rates of grade 3/4 rash (5.2\% vs. $0.3 \%)$ and fracture (2.7\% vs. $0.8 \%$ ) for apalutamide versus placebo. ${ }^{18}$ Rates of adverse events leading to treatment discontinuation were $9 \%$ for enzalutamide compared with $6 \%$ for placebo. There were higher rates of grade 3/4 hypertension (5\% vs. $2 \%$ ) and major adverse cardiovascular events (4\% vs. $2 \%$ ) with enzalutamide versus placebo. ${ }^{17}$ Both drugs were welltolerated overall, ${ }^{17,18,31}$ and the safety profile of enzalutamide was consistent with prior trials in the metastatic CRPC setting. ${ }^{32-35}$

\section{Discussion}

\section{Strengths and limitations}

The treatment algorithms for patients post-BCR (Fig. 1) and for nmCRPC (Fig. 2) represent practical and easy-to-follow tools to guide the management of non-metastatic PC. Although streamlined and helpful in guiding decision-making, especially among community clinicians, the algorithms have limitations. Inspired by current guidance, the algorithms reflect clinical opinion and consideration of recent evidence, but do not represent all available treatment options or the sum of all available evidence. Therefore, they should not be considered definitive or replacements for evidence-based clinical guidelines or consensus statements, but rather used to foster multi-disciplinary discussions of treatment options, including clinical trial enrolment, in light of individual disease characteristics and history, as well as patient preferences.

\section{Considerations for emerging therapies}

Historically, there has been a clinical need for effective therapeutic options for patients with nmCRPC. Recent data from the SPARTAN and PROSPER trials of apalutamide and enzalutamide, respectively, demonstrated that treatment prolonged metastasis-free survival in this setting, without detriment to overall quality of life. ${ }^{17,18}$ However, careful consideration should be given to use of these agents, as they involve increased side effects, high costs as well as a prolonged treatment duration. It also remains unclear whether the metastasis-free survival benefits will translate into improved overall survival. 


\section{PSAdt calculation}

The importance of accurate PSAdt calculation was highlighted in the SPARTAN and PROSPER trials, ${ }^{17,18}$ which required a PSAdt $<10$ months for eligibility. PSAdt calculation was performed using an online computation tool. ${ }^{36}$ For both trials, the median PSAdt was $<6$ months, ${ }^{17,18}$ and the majority of screening failures in the SPARTAN trial were due to the presence of metastatic disease, ${ }^{18}$ suggesting that early and accurate PSAdt assessments are important in treatment selection. Assessments should begin when PSA starts to rise and should include at least three PSA values, with at least one value $>2 \mathrm{ng} / \mathrm{ml}$.

\section{Role of novel imaging modalities}

The limitations in sensitivity and specificity of conventional imaging, including CT and BS, in detection of PC metastases are well-recognized. ${ }^{37,38}$ Incorporation of more sensitive PC-specific radiotracers, including ${ }^{18} \mathrm{~F}$-fluciclovine, ${ }^{11} \mathrm{C}$-choline and ${ }^{68} \mathrm{Ga}-\mathrm{PSMA}$, can improve early detection of recurrence, even at low PSA levels. ${ }^{24,25,39-41}$ Randomized trials are currently evaluating the role of PET scans in this patient population. ${ }^{42-45}$ Evidence now indicates a role for novel imaging in the management of local disease. ${ }^{19}$ The FALCON study, a prospective phase III trial of 85 patients post-BCR, demonstrated that 18F-fluciclovine PET/CT has substantial impact on clinical decisions, as $61.2 \%$ had a change in management strategy post-scan, ${ }^{19}$ while another prospective study of 188 patients post-BCR demonstrated that ${ }^{68} \mathrm{Ga}$-PSMA PET/CT detected tumor relapse in 165 patients (87.8\%), with a high level of sensitivity (98.8\%), specificity (100\%), and accuracy (98.8\%). ${ }^{24}$ Although conventional imaging via CT and BS remains standard practice in detecting metastatic disease, use of novel imaging modalities may be considered as they gain approval for use in Canada. In the nmCRPC setting, the phase III SPARTAN and the PROSPER trials established a benefit for apalutamide and enzalutamide, respectively, based on conventional imaging. ${ }^{17,18}$ It remains unclear how management recommendations will change, as newer imaging modalities detect metastatic disease in patients previously thought to have nmCRPC. 


\section{Patient-centred care, access to treatment and clinical trials}

Additional considerations in optimizing care for non-metastatic PC include ensuring equitable access to treatment, providing patient-centered and multidisciplinary care, and considering clinical trial participation at each treatment juncture. Management of PC is complex, and involves multiple clinical specialties, many treatment options, and may involve travel to multiple centres. This may be daunting for some patients, placing them at higher risk for experiencing disparities in care. ${ }^{46}$ Care plans should therefore include psychological and emotional support, facilitated access to treatment, and patient-centered decision making whenever possible. ${ }^{46,47}$ Clinical trials play a critical role in improving PC care and may represent important treatment options for patients, especially when access to emerging therapies is limited. Discussion of clinical trial options and dedicated efforts to remove demographic, socioeconomic and attitudinal factors that may hinder enrolment ${ }^{48,49}$ is an important part of optimal PC management.

\section{Summary}

The PC treatment landscape is continually evolving and presents an ongoing challenge for clinicians to consider and incorporate the latest systemic therapies and monitoring techniques. Management algorithms are practical and easy-to-use tools that can help streamline practice and inform multi-disciplinary management, leading to improved standards of care for non-metastatic PC.

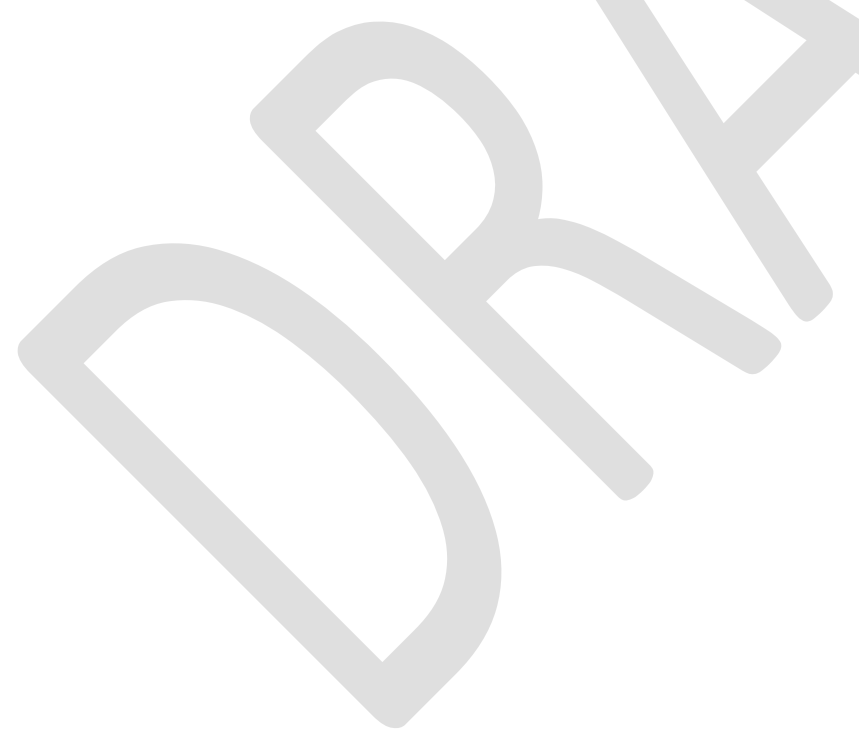




\section{References}

1. Canadian Cancer Society's Advisory Committee on Cancer Statistics. Canadian Caner Statistics 2017. Toronto, ON: Canadian Cancer Society 2017. Available at: http://www.cancer.ca/ /media/cancer.ca/CW/publications/Canadian\%20Cancer\%20Statis tics/Canadian-Cancer-Statistics-2017-EN.pdf; Accessed June 6, 2018.

2. Artibani W, Porcaro AB, De Marco V, et al. Management of Biochemical Recurrence after Primary Curative Treatment for Prostate Cancer: A Review. Urol Int. 2018;100:251-62.

3. Mottet N, van den Bergh RCN, Briers E, et al. EAU Guidelines: Prostate Cancer; 2018. Available at: https://uroweborg/guideline/prostate-cancer/Accessed April 17, 2018.

4. National Comprehensive Cancer Network. NCCN Clinical Practice Guidelines in Oncology (NCCN Guidelines ${ }^{\circledR}$ ): Prostate Cancer. Version 2.2018 — March 8, 2018. Available at: https://wwwnccnorg/store/login/loginaspx?ReturnURL=https://wwwnccnorg/professiona Is/physician_gls/pdf/prostatepdf Accessed April 15, 2018.2018.

5. Roach M, Hanks G, Thames H, et al. Defining biochemical failure following radiotherapy with or without hormonal therapy in men with clinically localized prostate cancer: recommendations of the RTOG-ASTRO Phoenix Consensus Conference. Int $J$ Radiat Oncol Biol Phys. 2006;65:965-74.

6. Cookson MS, Lowrance WT, Murad MH, et al. Castration-resistant prostate cancer: AUA guideline amendment. J Urol. 2015;193:491-9.

7. Gillessen S, Attard G, Beer TM, et al. Management of patients with advanced prostate cancer: the report of the Advanced Prostate Cancer Consensus Conference APCCC 2017. Eur Urol. 2018;73:178-211.

8. Loblaw A, Souter L, Canil C, et al. Follow-up Care for Survivors of Prostate CancerClinical Management: a Program in Evidence-Based Care Systematic Review and Clinical Practice Guideline. Clin Oncol. 2017;29:711-7.

9. Alberta Health Services. Clinical Practice Guideline: Prostate Cancer GU-004; 2015.Available at: https://www.albertahealthservices.ca/assets/info/hp/cancer/if-hpcancer-guide-gu004-prostate.pdf; Accessed March 18, 2018.

10. Catton C, Joshua A. Princess Margaret Cancer Centre Clinical Practice Guidelines: Prostate Cancer; 2015. Available at: http://wwwuhnca/PrincessMargaret/Health_Professionals/Programs_Departments/Genit ourinary_GU/Documents/CPG_GU_Prostatepdf Accessed March 18, 2018.

11. National Clinical Effectiveness Committee (Ireland). National Clinical Guideline for the Diagnosis, Staging and Treatment of Prostate Cancer; 2015. Available at: https://wwwhseie/eng/services/list/5/cancer/profinfo/guidelines/prostate/prostateguidelin epdf Accessed March 18, 2018.

12. Parker C, Gillessen S, Heidenreich A, et al. Cancer of the prostate: ESMO Clinical Practice Guidelines for diagnosis, treatment and follow-up. Ann Oncol. 2015;26:v69-v77. 
13. American Urological Association. Clinically Localized Prostate Cancer: AUA/ASTRO/SUO Guideline; 2017. Available at: http://wwwauanetorg/guidelines/clinically-localized-prostate-cancer-new-(aua/astro/suoguideline-2017) Accessed June 21, 2018.

14. Saad F, Chi KN, Finelli A, et al. The 2015 CUA-CUOG Guidelines for the management of castration-resistant prostate cancer (CRPC). Can Urol Assoc J. 2015;9:90.

15. Klotz L, Shayegan B, Guillemette C, et al. Testosterone suppression in the treatment of recurrent or metastatic prostate cancer-A Canadian consensus statement. Can Urol Assoc J. 2018;12:30.

16. Virgo KS, Rumble RB, Singer EA. Second-Line Hormonal Therapy for Men With Chemotherapy-Naïve Castration-Resistant Prostate Cancer: American Society of Clinical Oncology Provisional Clinical Opinion Summary. J Oncol Pract. 2017;13:459-61.

17. Hussain M, Fizazi K, Saad F, et al. Enzalutamide in Men with Nonmetastatic, CastrationResistant Prostate Cancer. N Engl J Med. 2018;378:2465-74.

18. Smith MR, Saad F, Chowdhury S, et al. Apalutamide treatment and metastasis-free survival in prostate cancer. N Engl J Med. 2018;378:1408-18.

19. Teoh EJ, Bottomley DM, Scarsbrook A, et al. The FALCON trial: Impact of 18Ffluciclovine PET/CT on clinical management choices for men with biochemically recurrent prostate cancer. J Clin Oncol. 2018;36:165.

20. Loizaga-Iriarte A, Rodriguez-Antolin A, Miñana B, et al. Castrate resistant prostate cancer. Consensus recommendations of the Spanish Association of Urology. Actas Urol Esp. 2017;41:141.

21. Golabek T, Belsey J, Drewa T, et al. Evidence-based recommendations on androgen deprivation therapy for localized and advanced prostate cancer. Cent European J Urol. 2016;69:131.

22. Torrecilla JL, Hervás A, Zapatero A, et al. Uroncor consensus statement: Management of biochemical recurrence after radical radiotherapy for prostate cancer: From biochemical failure to castration resistance. Rep Pract Oncol Radiother. 2015;20:259-72.

23. Droz J-P, Albrand G, Gillessen S, et al. Management of prostate cancer in elderly patients: recommendations of a task force of the International Society of Geriatric Oncology. Eur Urol. 2017;72:521-31.

24. Hamed MAG, Basha MAA, Ahmed H, et al. (68)Ga-PSMA PET/CT in Patients with Rising Prostatic-Specific Antigen After Definitive Treatment of Prostate Cancer: Detection Efficacy and Diagnostic accuracy. Acad Radiol. 2018.

25. Perera M, Papa N, Christidis D, et al. Sensitivity, Specificity, and Predictors of Positive 68Ga Prostate-specific Membrane Antigen Positron Emission Tomography in Advanced Prostate Cancer: A Systematic Review and Meta-analysis. Eur Urol. 2016;70:926-37.

26. Hoimes CJ, Kelly WK. Redefining hormone resistance in prostate cancer. Ther Adv Med Oncol. 2010;2:107-23.

27. Hou X, Flaig TW. Redefining hormone sensitive disease in advanced prostate cancer. Adv Urol. 2012;2012:978531. 
28. Smith MR, Antonarakis ES, Ryan CJ, et al. Phase 2 study of the safety and antitumor activity of apalutamide (ARN-509), a potent androgen receptor antagonist, in the highrisk nonmetastatic castration-resistant prostate cancer cohort. Eur Urol. 2016;70:963-70.

29. Smith MR, Kabbinavar F, Saad F, et al. Natural history of rising serum prostate-specific antigen in men with castrate nonmetastatic prostate cancer. J Clin Oncol. 2005;23:291825.

30. Smith MR, Saad F, Oudard S, et al. Denosumab and bone metastasis-free survival in men with nonmetastatic castration-resistant prostate cancer: Exploratory analyses by baseline prostate-specific antigen doubling time. J Clin Oncol. 2013;31:3800.

31. Beaver JA, Kluetz PG, Pazdur R. Metastasis-free Survival - A New End Point in Prostate Cancer Trials. N Engl J Med. 2018;378:2458-60.

32. Beer TM, Armstrong AJ, Rathkopf DE, et al. Enzalutamide in metastatic prostate cancer before chemotherapy. N Engl J Med. 2014;371:424-33.

33. Penson DF, Armstrong AJ, Concepcion R, et al. Enzalutamide Versus Bicalutamide in Castration-Resistant Prostate Cancer: The STRIVE Trial. J Clin Oncol. 2016;34:2098106.

34. Scher HI, Fizazi K, Saad F, et al. Increased survival with enzalutamide in prostate cancer after chemotherapy. $N$ Engl J Med. 2012;367:1187-97.

35. Shore ND, Chowdhury S, Villers A, et al. Efficacy and safety of enzalutamide versus bicalutamide for patients with metastatic prostate cancer (TERRAIN): a randomised, double-blind, phase 2 study. Lancet Oncol. 2016;17:153-63.

36. Memorial Sloan Kettering Cancer Center PSA Doubling Time Calculator. Available at: https://wwwmskccorg/nomograms/prostate/psa_doubling_time; Accessed July 5, 2018.

37. Cook GJR, Azad G, Padhani AR. Bone imaging in prostate cancer: the evolving roles of nuclear medicine and radiology. Clin Transl Imaging. 2016;4:439-47.

38. Leung D, Krishnamoorthy S, Schwartz L, et al. Imaging approaches with advanced prostate cancer: techniques and timing. Can J Urol. 2014;21:42-7.

39. Evangelista L, Briganti A, Fanti S, et al. New Clinical Indications for 18F/11C-choline, New Tracers for Positron Emission Tomography and a Promising Hybrid Device for Prostate Cancer Staging: A Systematic Review of the Literature. Eur Urol. 2016;70:16175.

40. Sobol I, Zaid HB, Haloi R, et al. Contemporary Mapping of Post-Prostatectomy Prostate Cancer Relapse with 11C-Choline Positron Emission Tomography and Multiparametric Magnetic Resonance Imaging. J Urol. 2017;197:129-34.

41. Parker WP, Evans JD, Stish BJ, et al. Patterns of Recurrence After Postprostatectomy Fossa Radiation Therapy Identified by C-11 Choline Positron Emission Tomography/Computed Tomography. Int J Radiat Oncol Biol Phys. 2017;97:526-35.

42. Cihoric N, Vlaskou Badra E, Tsikkinis A, et al. Clinical trials involving positron emission tomography and prostate cancer: an analysis of the ClinicalTrials.gov database. Radiat Oncol. 2018;13:113.

43. Bouchelouche K, Choyke PL. Advances in prostate-specific membrane antigen PET of prostate cancer. Curr Opin Oncol. 2018;30:189-96. 
44. Hope TA, Afshar-Oromieh A, Eiber M, et al. Imaging Prostate Cancer With ProstateSpecific Membrane Antigen PET/CT and PET/MRI: Current and Future Applications. AJR Am J Roentgenol. 2018:1-9.

45. Hofman MS, Murphy DG, Williams SG, et al. A prospective randomized multicentre study of the impact of gallium-68 prostate-specific membrane antigen (PSMA) PET/CT imaging for staging high-risk prostate cancer prior to curative-intent surgery or radiotherapy (proPSMA study): clinical trial protocol. BJU Int. 2018.

46. Ahmed S, Shahid RK. Disparity in cancer care: a Canadian perspective. Curr Oncol. 2012;19:e376-e82.

47. Biddy R, Griffin C, Johnson N, et al. Person-Centred Care Guideline. Cancer Care Ontaio and Program in Evidence-Based Care 2015; Available at: https://archive.cancercare.on.ca/common/pages/UserFile.aspx?fileId=340815; Accessed on June 27, 2018.

48. Manne S, Kashy D, Albrecht T, et al. Attitudinal barriers to participation in oncology clinical trials: factor analysis and correlates of barriers. Eur J Cancer Care (Engl). 2015;24:28-38.

49. Unger JM, Cook E, Tai E, et al. Role of Clinical Trial Participation in Cancer Research: Barriers, Evidence, and Strategies. American Society of Clinical Oncology educational book; ASCO Annual Meeting. 2016;35:185-98.

50. Crook JM, O'Callaghan CJ, Duncan G, et al. Intermittent androgen suppression for rising PSA level after radiotherapy. N Engl J Med. 2012;367:895-903. 


\section{Figures and Tables}

Fig. 1. Management algorithm for prostate cancer patients with a biochemical recurrence after radical local therapy. This algorithm does not address other aspects of care such as bone health and cardiovascular health. ${ }^{1}$ Clinicians should consider a lower PSA threshold when there is no prostate in-situ; ${ }^{2}$ PSA doubling time can be easily calculated using an online calculator:

https://www.mskcc.org/nomograms/prostate/psa_doubling_time; ${ }^{3}$ If on iADT and PSA nadir $\geq 1$ $\mathrm{ng} / \mathrm{ml}$ or off-treatment interval $<10$ months consider switching to cADT; clinicians should also consider switching from iADT to cADT if patients do not achieve a PSA nadir of at least $1 \mathrm{ng} / \mathrm{ml}$ after 6 months of iADT; 50 Lower testosterone levels (testosterone $\leq 0.7 \mathrm{nmol} / \mathrm{L}$ ) have been associated with improved outcomes; secondary hormonal manipulations (switch ADT or add antiandrogen) may be considered if testosterone is $>0.7 \mathrm{nmol} / \mathrm{L} .{ }^{15} \mathrm{AA}$ : antiandrogen; (c/i)ADT: (continuous/intermittent) androgen-deprivation therapy; BS: bone scan; CRPC: castration-resistant prostate cancer; CT: computed tomography; dt: doubling time; mets: metastases; nm: nonmetastatic; PSA: prostate-specific antigen; pts: patients; RP: radical prostatectomy; RT: radiation therapy; test: testosterone.

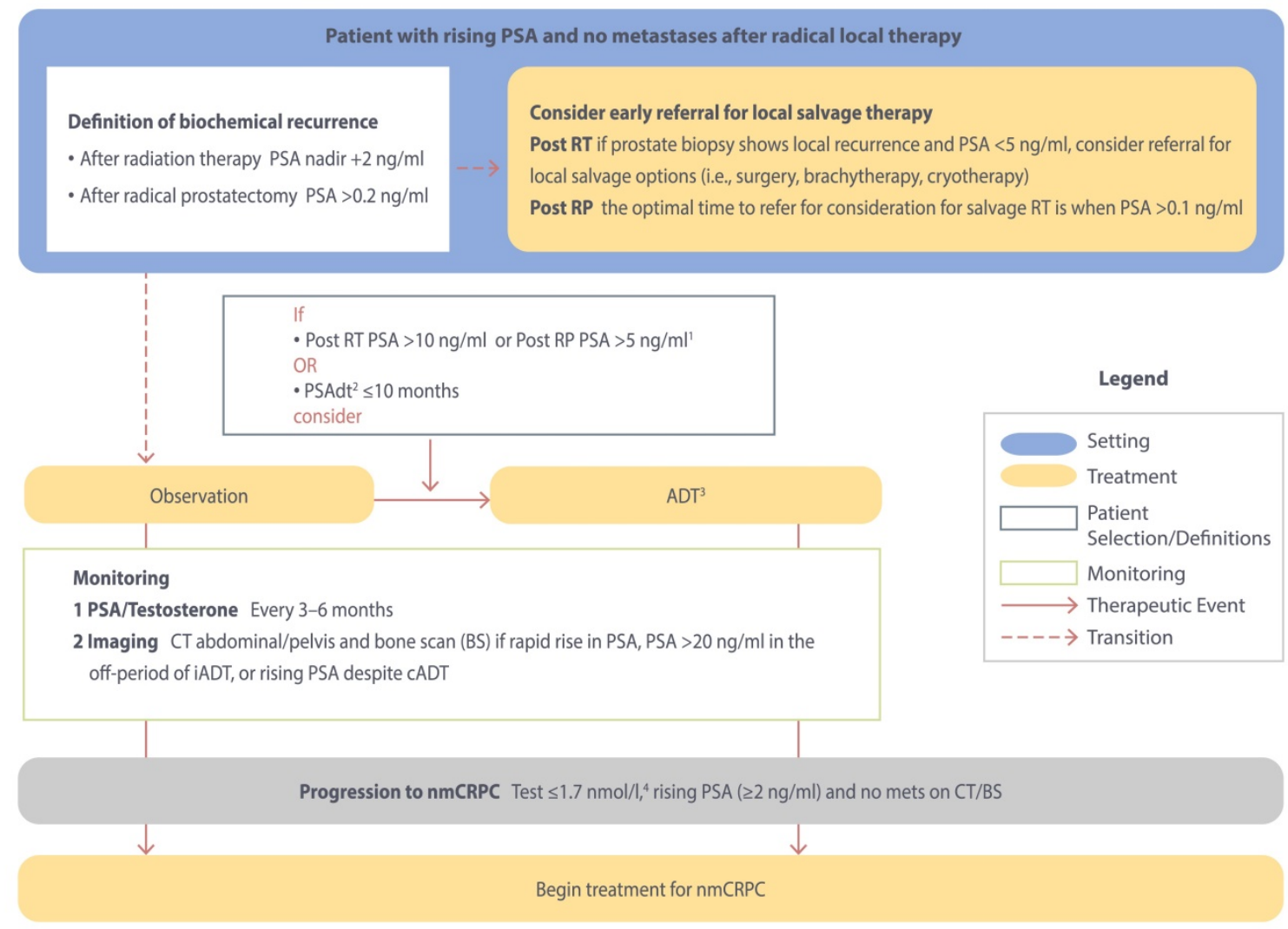


Fig. 2. Management algorithm for nmCRPC. This algorithm does not address other aspects of care such as bone health and cardiovascular health. ${ }^{1}$ An individualized approach to treatment selection should take into consideration the pros and cons of therapy, as well as patient characteristics and preference; ${ }^{2}$ There is emergent evidence for the benefit of local therapy in select nmCRPC patients; ${ }^{3}$ PSA doubling time can be easily calculated using an online calculator: https://www.mskcc.org/nomograms/prostate/psa_doubling_time; ${ }^{4}$ Health Canada has approved apalutamide, and enzalutamide approval is pending; both treatments have shown a statistically significant improvement in the primary end-point of metastasis-free survival in phase 3 trials; overall survival data is not yet mature. AA: anti-androgen; (c/i)ADT:

(continuous/intermittent) androgen-deprivation therapy; BS: bone scan; CRPC: castrationresistant prostate cancer; CT: computed tomography; dt: doubling time; mets: metastases; nm: non-metastatic; PSA: prostate-specific antigen; pts: patients; RP: radical prostatectomy; RT: radiation therapy; test: testosterone.

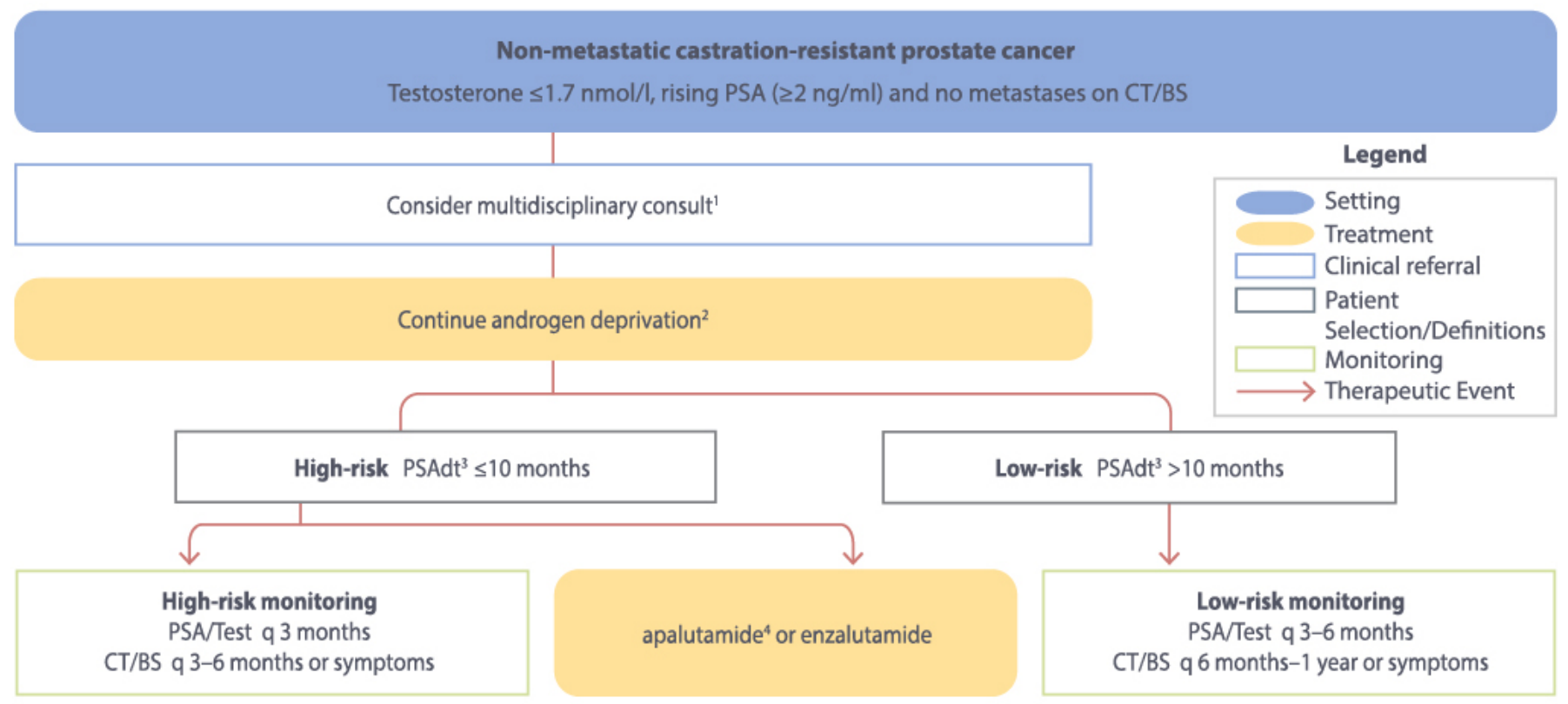




\begin{tabular}{|l|c|c|}
\hline \multicolumn{2}{|l|}{ Table 1. Guidelines, consensus-based guidance, and original research considered in } \\
development of the algorithms & Year & Region \\
\hline Guidelines and consensus statements & 2018 & Canadian \\
\hline Testosterone suppression Canadian consensus statement & 2018 & American \\
\hline NCCN prostate cancer guidelines & 2018 & European \\
\hline EAU-ESTRO-ESUR-SIOG guidelines on prostate cancer & 2017 & International \\
\hline Advanced Prostate Cancer Consensus Conference (APCCC) & 2017 & American \\
\hline ASCO CRPC provisional clinical opinion & 2015 & Canadian \\
\hline CUA-CUOG CRPC guidelines & 2015 & European \\
\hline ESMO clinical practice guidelines on prostate cancer & 2015 & American \\
\hline AUA CRPC guidelines & 2018 & Year \\
\hline Original research on treatment for nmCRPC & 2018 & \\
\hline FALCON (Role of F-fluciclovine PET/CT) & & \\
\hline SPARTAN (apalutamide) & & \\
\hline PROSPER (enzalutamide) & 2018 \\
\hline
\end{tabular}

ASCO: American Society of Clinical Oncology; AUA: American Urological Association; CUACUOG: Canadian Urological Association-Canadian Urologic Oncology Group; CRPC: castration-resistant prostate cancer; EAU-ESTRO-ESUR-SIOG: European Association of Urology-European Society for Radiotherapy \& Oncology- European Society of Urogenital Radiology-International Society of Geriatric Oncology; FALCON: Fluciclovine (18F) PET/CT in biochemicAL reCurrence Of Prostate caNcer; NCCN: National Comprehensive Cancer Network; PET/CT: positron emission tomography/computed tomography; PROSPER: Safety and Efficacy Study of Enzalutamide in Patients With Non-metastatic Castration-Resistant Prostate Cancer; SPARTAN: A Study of Apalutamide (ARN-509) in Men With Non-Metastatic CastrationResistant Prostate Cancer. 